\title{
Stably asymptotic average shadowing property and dominated splitting
}

Manseob Lee

Correspondence: Imsds@mokwon. ac.kr

Department of Mathematics, Mokwon University, Daejeon 302729, Korea

\section{Abstract}

Let $f$ be a diffeomorphism of a closed $n$-dimensional $C^{\infty}$ manifold. In this article, we show that $C^{1}$-generically, if $f$ has the $C^{1}$-stably asymptotic average shadowing property on a closed set then it admits a dominated splitting.

Mathematical Subject Classification: 34D05; 37C20; 37D30.

Keywords: asymptotic average shadowing, dominated splitting, transitive, chain transitive

\section{Introduction}

The notion of the pseudo-orbits very often appears in several branches of the modern theory of dynamical system. For instance, the pseudo-orbit property (shadowing property) usually plays an important role in stability theory In this article, we consider the asymptotic average shadowing property, which was introduced in $\mathrm{Gu}$ [1], is a special version of the shadowing property We find a relation between the stably asymptotic average shadowing property (on manifold) and the dominated splitting structure on the vector bundle. In differentiable dynamical system, dominated splitting on the vector bundle is a nature generalization of hyperbolicity and is investigated by many mathematicians [2-11].

Here we denote $M$ a closed $n$-dimensional smooth manifold, and let $\operatorname{Diff}(M)$ be the space of diffeomorphisms of $M$ endowed with the $C^{1}$-topology. Denote by $d$ the distance on $M$ induced from a Riemannian metric $\|\cdot\|$ on the tangent bundle TM. Let $f \in$ Diff (M). A sequence $\left\{x_{i}\right\}_{i=-\infty}^{\infty}$ in $M$ is called an asymptotic average pseudo orbit of $f$ if

$$
\lim _{n \rightarrow \infty} \frac{1}{2 n} \sum_{i=-n}^{n-1} d\left(f\left(x_{i}\right), x_{i+1}\right)=0 .
$$

An asymptotic average pseudo orbit $\left\{x_{i}\right\}_{i \in \mathrm{Z}}$ is said to be asymptotically shadowed in average by the point $z$ if

$$
\lim _{n \rightarrow \infty} \frac{1}{2 n} \sum_{i=-n}^{n-1} d\left(f^{i}(z),\left(x_{i}\right)=0 .\right.
$$

Given an invariant set $\Lambda$ of $f$, we say $f$ has the asymptotic average shadowing property on $\Lambda$ if for any asymptotic pseudo orbit $\left\{x_{i}\right\}_{i \in Z}$, there exist a point $z \in \Lambda$ which asymptotically shadows $\left\{x_{i}\right\}_{i \in Z}$. 
Let $f \in \operatorname{Diff}(M)$, and let $\Lambda$ be a closed $f$-invariant set. We say that $\Lambda$ is locally maximal if there is a compact neighborhood $U$ of $\Lambda$ such that $n_{n \in \mathbb{N}} f^{n}(U)=\Lambda$. Now we can introduce a notion of $C^{1}$-stably the asymptotic average shadowing property on a locally maximal invariant set.

Definition 1.1 Let $\Lambda$ be a locally maximal invariant set of $f \in \operatorname{Diff}(M)$. We say that $f$ has the $C^{1}$-stably asymptotic average shadowing property on $\Lambda$, ( or $\Lambda$ is $C^{1}$-stably asymptotic average shadowable with respect to $f$ ) if there are a compact neighborhood $U$ of $f$ and a $C^{1}$-neighborhood $\mathcal{U}(f)$ of $f$ such that $\Lambda=\Lambda_{f}(U)=\Lambda_{n \in Z} f^{n}(U)$ (locally maximal), and for any $g \in \mathcal{U}(f),\left.g\right|_{\Lambda_{g}(U)}$ has the asymptotic average shadowing property, where $\Lambda_{g}(U)=\Lambda_{n \in Z} g^{n}(U)$ is the continuation of $\Lambda$.

Let $\Lambda \subset M$ be an $f$-invariant closed set. We say that $\Lambda$ admits a dominated splitting if the tangent bundle $T_{\Lambda} M$ has a continuous $D f$-invariant splitting $E \oplus F$ and there exist constants $C>0$ and $0<\lambda<1$ such that

$$
\left\|D_{x} f^{n}\left|E_{(x)}\|\cdot\| D_{x} f^{-n}\right|_{F\left(f^{n}(x)\right)}\right\| \leq C \lambda^{n}
$$

for all $x \in \Lambda$ and $n \geq 0$.

The following remark gives an equivalent definition of dominated splitting.

Remark 1.2 Let $\Lambda$ be a closed f-invariant set. A splitting $T_{\Lambda} M=E \oplus F$ is called a $l$ dominated splitting for a positive integer $l$ if $E$ and $F$ are Df-invariant and

$$
\left\|\left.D f^{l}\right|_{E(x)}\right\| / m\left(\left.D f^{l}\right|_{F(x)}\right) \leq \frac{1}{2}
$$

for all $x \in \Lambda$, where $m(A)=\inf \{\|A v\|:\|v\|=1\}$ denotes the minimum norm of a linear map $A$.

Now we can state main results of this article.

Theorem 1.3 Let $\Lambda$ be a closed set of $f \in \operatorname{Diff}(M)$. Then $C^{1}$-generically, if $f$ has the $C^{1}$-stably asymptotic average shadowing property on $\Lambda$ then it admits a dominated splitting.

Theorem 1.4 Let $\Lambda$ be a transitive set. If $f$ has the $C^{1}$-stably asymptotic average shadowing property on $\Lambda$ then it admits a dominated splitting.

\section{Proof of theorems}

Theorems 1.3 and 1.4 are all base on the following proposition:

Proposition 2.1 Let $\Lambda$ be a closed locally manximal invariant set of $f$, if $f$ has the $C^{1}$ stably asymptotic average shadowing property on $\Lambda$, and there exist a sequence $g_{n}$ goes to $f$ and periodic orbits $P_{n}$ of $g_{n}$ which converges to $\Lambda$ in Hausdorff limits, then $\Lambda$ admits a dominated splitting.

Firstly, we give the notation of pre-sink (resp. pre-source) which prevent the stably asymptotic average shadowing property. A periodic point $p$ of $f$ is called a pre-sink (resp. pre-source) if $D f^{\pi(p)}(p)$ has a multiplicity one eigenvalue with modulus 1 and the other eigenvalues has norm strictly less than 1 (resp. bigger than 1 ).

Lemma 2.2 Let $\Lambda$ be a closed set of $f$. Suppose that $f_{\mid \Lambda}$ has the $C^{1}$-stably asymptotic average shadowing property. Let $U$ and $\mathcal{U}(f)$ be given in the Definition 1.1, then for any $g \in \mathcal{U}(f), g$ has neither pre-sink nor pre-sources with the orbit staying in $U$.

Proof. We prove the lemma by contradiction. Assume that there is $g \in \mathcal{U}(f)$ such that $g$ has a pre-sink $p$ with $\operatorname{Orb}(p) \subset U$. 
By the Franks' Lemma, we can linearize $g$ at $p$ with respect to the exponential coordinates $\exp _{p}$, i.e., after an arbitrarily small perturbation, we can get a diffeomorphism $g_{1} \in \mathcal{U}(f)$ such that there is $\epsilon_{1}>0$ small enough with $B_{\varepsilon_{1}}(\operatorname{Orb}(p)) \subset U$ such that

$$
\left.g_{1}\right|_{B_{\varepsilon_{1}}\left(g^{i}(p)\right)}=\left.\exp _{g^{i+1}(p)} \circ D_{g^{i}(p)} g \circ \exp _{g^{i}(p)}^{-1}\right|_{B_{\varepsilon_{1}}\left(g^{i}(p)\right)^{\prime}}
$$

for any $0 \leq i \leq \pi(p)-1$.

Since $p$ is pre-sink of $g, D_{p} g^{\pi(p)}$ has a multiplicity one eigenvalue such that $|\lambda|=1$ and other eigenvalues of $D_{p} g^{\pi(p)}$ have moduli less than 1 . Denote by $E_{p}^{c}$ the eigenspace corresponding to $\lambda$, and $E_{p}^{s}$ the eigenspace corresponding to the eigenvalues with modulus less than 1 . Thus $T_{p} M=E_{p}^{c} \oplus E_{p}^{s}$. If $\lambda \in \mathbb{R}$ then $\operatorname{dim} E_{p}^{c}=1$, and if $\lambda \in \mathbb{C}$ then $\operatorname{dim} E_{p}^{c}=2$.

At first, we consider the case $\operatorname{dim} E_{p}^{c}=1$. For simplicity, we suppose that $\lambda=1$, and $g_{1}^{\pi(p)}(p)=p$. The case of $\lambda=-1$ can be proved similarly. Since the eigenvalue $\lambda=1$, there is a small arc $\mathcal{I}_{p} \subset B_{\varepsilon_{1}}(p) \cap \exp _{p}\left(E_{p}^{c}\left(\varepsilon_{1}\right)\right)$ centered at $p$ such that $\left.g_{1}^{\pi(p)}\right|_{\mathcal{I}_{p}}$ is the identity map. Here $E_{p}^{c}\left(\varepsilon_{1}\right)$ is the $\epsilon_{1}$-ball in $E_{p}^{c}$ center at the origin $O_{p}$.

There exist $D>0$ such that for any $z \in B_{D}(p)$, there exists $x \in \mathcal{I}_{p}$ such that $g_{1}^{n \pi(p)}(z) \rightarrow x$ as $n \rightarrow \infty$. Take two distinct points $a, b \in \mathcal{I}_{p}$ such that $d(a, b)=D / 4$.

We construct an asymptotic average pseudo orbit of $g_{1}$ as follows.

$$
\begin{aligned}
& x_{-i}=g_{1}^{-i} a, \\
& x_{0}=a, x_{1}=g_{1}(a), \ldots, x_{\pi(p)-1}=g_{1}^{\pi(p)-1} a, x_{\pi(p)}=b, \ldots, \\
& x_{\left(2^{k}-2\right) \pi(p)}=a, x_{\left(2^{k}-2\right) \pi(p)+1}=g_{1}(a), \ldots, x_{\left(2^{k}+2^{k-1}-2\right) \pi(p)-1}=g_{1}^{-1} a, \\
& x_{\left(2^{k}+2^{k-1}-2\right) \pi(p)}=b, \ldots, x_{\left(2^{k+1}-2\right) \pi(p)-1}=g_{1}^{-1}(b), \ldots
\end{aligned}
$$

One can easily check that $\xi=\left\{x_{i}\right\}_{i \in Z}$ is an asymptotic average pseudo orbit of $g_{1}$.

Since $g_{1}$ has the asymptotic average shadowing property on $\Lambda_{g_{1}}(U)$, we can find a point $z$ such that the point $z$ is shadows $\xi=\left\{x_{i}\right\}_{i \in Z}$ in asymptotic average, i.e.,

$$
\lim _{n \rightarrow \infty} \frac{1}{2 n} \sum_{i=-n}^{n-1} d\left(g_{1}^{i}(z), x_{i}\right)=0 .
$$

It is easy to see that there is $n_{0}>0$ such that $g_{1}^{n_{0}}(z) \in B_{D}(p)$. Hence there exists a point $x \in \mathcal{I}_{p}$ such that $g_{1}^{n \pi(p)+n_{0}}(z) \rightarrow x$, as $n \rightarrow \infty$. From the choice of $a, b$ and the fact that $\left.g_{1}^{\pi(p)}\right|_{\mathcal{I}_{p}}=I d$, we have

$$
\lim _{n \rightarrow \infty} \frac{1}{n} \sum_{i=0}^{n-1} d\left(g_{1}^{i}(z), x_{i}\right)=\lim _{n \rightarrow \infty} \frac{1}{n} \sum_{i=0}^{n-1} d\left(g_{1}^{i-n 0}(x), x_{i}\right)>0 .
$$

This is a contradiction.

Finally, we consider the case $\operatorname{dim} E_{p}^{c}=2$. There is a disk $\mathcal{D}_{p} \subset B_{\varepsilon_{1}}(p) \cap \exp _{p}\left(E_{p}^{c}\left(\varepsilon_{1}\right)\right)$ centered at $p$ such that $\left.g_{1}^{\pi(p)}\right|_{\mathcal{D}_{p}}$ is a rotation. Note that $\mathcal{D}$ consists of $g_{1}^{\pi(p)}$-invariant circles. We take $a$ and $b$ in different circles. Then by similar 
arguments as above, we get the contradiction. We omit the details and finish the proof here.

Let $G L(n)$ be the group of linear isomorphisms of $\mathbb{R}^{n}$. A sequence $\xi: \mathbb{Z} \rightarrow G L(n)$ is called periodic if there is $k>0$ such that $\xi_{j+k}=\xi_{j}$ for $k \in \mathbb{Z}$. We call a finite subset $\mathcal{A}=\left\{\xi_{i}: 0 \leq i \leq k-1\right\} \subset G L(n)$ is a periodic family with period $k$. For a periodic family $\mathcal{A}=\left\{\xi_{i}: 0 \leq i \leq n-1\right\}$, we denote $\mathcal{C}_{\mathcal{A}}=\xi_{n-1} \circ \xi_{n-2} \circ \cdots \circ \xi_{0}$.

Definition 2.3 We say that the periodic family $\mathcal{A}=\left\{\xi_{i}: 0 \leq i \leq n-1\right\}$ admits an ldominated splitting, if there is a splitting $\mathbb{R}^{n}=E \oplus F$ which satisfies:

(a) $E$ and $F$ are $\mathcal{C}_{\mathcal{A}}$ invariant, i.e., $\mathcal{C}_{\mathcal{A}}(E)=E$ and $\mathcal{C}_{\mathcal{A}}(F)=F$,

(b) For any $k=0,1,2, \ldots$,

$$
\frac{\left\|\left.\xi_{k+l-1} \circ \cdots \circ \xi_{k+1} \circ \xi_{k}\right|_{E_{k}}\right\|}{m\left(\left.\xi_{k+l-1} \circ \cdots \circ \xi_{k 1} \circ \xi_{k}\right|_{F_{k}}\right)} \leq \frac{1}{2}
$$

where $E_{k}=\xi_{k-1} \circ \xi_{k-2} \circ \ldots \circ \xi_{0}(E)$ and $F_{k}=\xi_{k-1} \circ \xi_{k-2} \circ \ldots \circ \xi_{0}(F)$.

We know the following theorems for periodic family from [4] which is useful for our result.

Theorem 2.4 Given any $\epsilon>0$ and $K>0$, there is positive integers $n_{2} \geq 0$ and $l \geq 0$ which satisfies the following property: given any periodic family $\mathcal{A}=\left\{\xi_{i}: 0 \leq i \leq n-1\right\}$ which satisfies the period $n \geq n_{2}$ and $\max \left\{\left\|\xi_{i}\right\|,\left\|\xi_{i}^{-1}\right\|\right\} \leq K$, for all $i=0,1, \ldots, n-1$, if $\mathcal{A}$ does not admits any l-dominated splitting, then one can find a periodic family $\mathcal{B}=\left\{\zeta_{0}, \zeta_{1}, \ldots, \zeta_{n-1}\right\}$ such that $\max \left\{\left\|\zeta_{i}-\xi_{i}\right\|,\left\|\zeta_{i}^{-1}-\xi_{i}^{-1}\right\|\right\}<\varepsilon$ for any $i=0,1, \ldots, n-1$, and $\operatorname{det}\left(\mathcal{C}_{\mathcal{A}}\right)=\operatorname{det}\left(\mathcal{C}_{\mathcal{B}}\right)$ and the eigenvalues of $\mathcal{C}_{\mathcal{B}}$ are all real, and have same modulus.

To prove Theorem 2.4, we need another lemma about uniformly contracting family. Let $\mathcal{A}=\left\{\xi_{i}: 0 \leq i \leq k-1\right\} \subset G L(n)$ be a periodic family. We say the sequence $\mathcal{A}$ is uniformly contracting family if there is a constant $\delta>0$ such that for any $\delta$-perturbation of $\mathcal{A}$ are sink, i.e., for any $\mathcal{B}=\left\{\xi_{i}: 0 \leq i \leq k-1\right\}$ with $\left\|\zeta_{i}-\zeta_{i}\right\|<\delta$, all eigenvalue of $\mathcal{C}_{\mathcal{B}}$ have moduli less than 1 . Similarly, we can define the uniformly expanding periodic family. The following theorem is well known.

Theorem 2.5 [12] For any $\delta>0$ and $K>0$, there are constants $C>0,0<\lambda<1$ and positive integer $m$ such that if $\mathcal{A}=\left\{A_{0}, A_{1}, \ldots, A_{n-1}\right\}$ is a uniformly contracting periodic family which satisfies

$$
\max \left\{\left\|A_{i}\right\|,\left\|A_{i}^{-1}\right\|\right\}<K
$$

for any $i=0,1, \ldots, n-1$ and $n>m$, then

$$
\prod_{j=0}^{k-1}\left\|\prod_{i=0}^{m-1} A_{i+m j}\right\| \leq C \lambda^{k},
$$

where $k=[n / m]$.

Now we return to our main proposition, the Proposition 2.1. Let $P_{n}$ be given as in 
Proposition 2.1. Choose $p_{n} \in P_{n}$, then we get a linear map sequence

$$
\mathcal{A}_{n}=\left\{D_{p_{n}} f, D_{f\left(p_{n}\right)} f, \ldots, D_{f \pi\left(p_{n}\right)-1}\left(p_{n}\right) f\right\} .
$$

Lemma 2.6 [[10], Lemma 3.2.] If $\Lambda$ is not a periodic orbit and $\mathcal{A}_{n}$ is given in above. Then for any $\epsilon>0$ there exists an $n_{0}(\epsilon)>0$ such that for any $n>n_{0}(\epsilon), \mathcal{A}_{n}$ is neither $\epsilon$-uniformly contracting nor $\epsilon$-uniformly expanding.

Since the proof is essentially the same as that of [10], we omit the proof here. From the above lemma and main conclusion of [4], one can get the following lemma. The proof of the following can be found in [10].

Lemma 2.7 [[10], Lemma 3.3.] Let $\Lambda, g_{n}$ and $P_{n}$ be given as in the assumption of Proposition 2.1. Then for any $\epsilon>0$ there are $n(\epsilon), l(\epsilon)>0$ such that for any $n>n(\epsilon)$ if $P_{n}$ does not admit an $l(\epsilon)$ dominated splitting, then one can find $g_{n}^{\prime} C^{1} \epsilon$-close $g_{n}$ and preserving the orbit of $P_{n}$ such that $P_{n}$ is pre-sink or pre-source respecting $g_{n}^{\prime}$.

From the above lemmas and the next property of dominated splitting, we can get Proposition 2.1.

Lemma 2.8 [[3], Lemma 1.4.] Let $g_{n}$ converges to $f$ and if $\Lambda_{n}$ be a closed $g_{n}$-invariant set such that the Hausdorff limit of $\Lambda_{n}$ equal to $\Lambda$. If $\Lambda_{g_{n}}(U)$ admits a l-dominated splitting respecting $g_{n}$, then $\Lambda$ admits an l-dominated splitting respecting $f$.

Now we can get our Theorems 1.3 and 1.4, Theorem 1.3 follows two results:

Lemma 2.9 [1,13] Let $\Lambda$ be a closed set of $f \in \operatorname{Diff}(M)$. If $f$ has the asymptotic average shadowing property on $\Lambda$ then $\Lambda$ is a chain transitive set.

The following Lemma is in [14].

Lemma 2.10 There is a residual set $\mathcal{G} \subset \operatorname{Diff}(M)$ such that for any $f \in \mathcal{G}$, a compact $f$-invariant set $\Lambda$ is a chain transitive set if and if $\Lambda$ is a sequence $\left\{P_{n}\right\}$ of periodic orbits of $f$ with the Hausdorff topology.

Theorem 1.4 follows the result:

Lemma 2.11 [[11], Corollary 2.7.1.] Let $\Lambda$ be a transitive set. Then there are a sequence $\left\{g_{n}\right\}$ of diffeomorphism and a sequence $\left\{P_{n}\right\}$ of periodic orbits of $g_{n}$ with period $\pi\left(P_{n}\right) \rightarrow \infty$ such that $g_{n} \rightarrow f$ in the $C^{\mathrm{l}}$-topology and $P_{n} \rightarrow{ }_{H} \Lambda$ as $n \rightarrow \infty$, where $\rightarrow{ }_{H}$ is the Hausdorff limit, and $\pi\left(P_{n}\right)$ is the period of $P_{n}$.

\footnotetext{
Acknowledgements

The author wishes to express his deepest appreciation to the referee for his careful reading of the manuscript, critical comments and many valuable suggestions; the author want to thank Prof. W. Xiao for helpful conversations. This study was supported by the Basic Science Research Program through the National Research Foundation of Korea (NRF) funded by the Ministry of Education, Science and Technology (No. 2011-0007649).

Competing interests

The author declares that they have no competing interests.

Received: 22 October 2011 Accepted: 5 March 2012 Published: 5 March 2012

References

1. Gu, R: The asymptotic average shadowing property and transitivity. Nonlinear Anal. 67, 1680-1689 (2007). doi:10.1016/j. na.2006.07.040

2. Abdenur, F, Bonatti, C, Corvisier, S: Global dominated splitting and the $C^{1}$-newhouse phenomenon. Proc Am Math Soc. 134, 2229-2237 (2006). doi:10.1090/S0002-9939-06-08445-0

3. Bonatti, C, Díaz, LJ, Pujals, E: A C Cl-generic dichotomy for diffeomorphisms: weak forms of hyperbolicity or infinitely many sinks or sources. Ann Math. 158, 187-222 (2003)

4. Bonatti, C, Gourmelon, N, Vivier, T: Perturbations of the derivative along periodic orbits. Ergodi Th Dyn Syst. 26, 1307-1337 (2006). doi:10.1017/S0143385706000253

5. Gan, S, Sakai, K, Wen, L: C ${ }^{1}$-stably weakly shadowing homoclinic classes. 27, 205-216 (2010)
} 
6. Lee, M: Transitive sets with dominated splitting. J Chung Math Soc. 23, 65-71 (2010)

7. Lee, M: Dominated splitting with stably expansive. J Chung Math Soc. 18, 285-291 (2011)

8. Lee, k, Lee, M: Stably inverse shadowable transitive sets and dominated splitting. Proc Am Math Soc. 140, 271-226 (2012)

9. Lee, k, Lee, M, Anh, J: Stably periodic shadowing and dominated splitting. J Chung Math Soc. 24, 735-743 (2011)

10. Lee, $k, L u, G, W e n, X: C^{1}$-stably weak shadowing property of chain transitive sets (preprint).

11. Yang, D: Stably weakly shadowing transitive sets and dominated splitting. Proc Am Math Soc. 139, 2747-2751 (2011). doi:10.1090/50002-9939-2011-10699-3

12. Mãné, R: An ergodic closing lemma. Ann Math. 116, 503-540 (1982). doi:10.2307/2007021

13. Honary, B, Bahabadi, A: Asymptotic average shadowing property on compact metric spaces. Nonlinear Anal. 69, 2857-2863 (2008). doi:10.1016/j.na.2007.08.058

14. Corvisier, S: Periodic orbit and chain transitive sets of $C^{1}$-diffeomorphisms. Publ Math Inst Hautes tudes Sci. 104, 87-141 (2006)

doi:10.1186/1687-1847-2012-25

Cite this article as: Lee: Stably asymptotic average shadowing property and dominated splitting. Advances in

Difference Equations 2012 2012:25.

\section{Submit your manuscript to a SpringerOpen ${ }^{\circ}$} journal and benefit from:

- Convenient online submission

- Rigorous peer review

- Immediate publication on acceptance

- Open access: articles freely available online

- High visibility within the field

- Retaining the copyright to your article

Submit your next manuscript at $\gg$ springeropen.com 